\title{
Enantioseparation of Nadifloxacin by High performance liquid Chromatography
}

\author{
Surendra Dutt Sharma, Gaurav Singh* \\ Analy tical Research Laboratory, School of Sciences.IFTM University Campus, Moradabad, 244102, Uttar Pradesh, India
}

\begin{abstract}
A rapid isocratic chiral HPLC method has been developed for the separation of R-Nadifloxacin from S-Nadifloxacin. Good resolution viz. Rs> 4.0 between R- and S- forms of Nadifloxacin was achieved by RP-HPLC using endcappedC18 stationary phase and chiral mobile phase. Chira lity to the mobile phase was imparted with addition of $\beta$-CD in phosphate buffer with EDTA.Colu mn temperature was $45^{\circ} \mathrm{C}$ and flow rate was kept $1.5 \mathrm{~mL} \mathrm{~min}^{-1}$. The elution was monitored by UV-vis detector at $\lambda-290 \mathrm{~nm}$. The calibration curve showed excellent linearity over concentration range $0.040-20 \mu \mathrm{g}$ $\mathrm{mL}^{-1}$. This method was further used to determine the amount of $\mathrm{R}$-Nadiflo xac in in pure and active pharmaceutical ingredient of S-Nadifloxacin and is capable to quantitate and detect R-Nadifloxacin to the levels of $0.040 \mu \mathrm{gL}^{-1}$ and $0.020 \mu \mathrm{g} \mathrm{mL}$ respectively. The average recovery of R-Nadiflo xac in was $99.09 \%$. The method is better than the already reported one for the enantioseparation of the Nadiflo xacin.
\end{abstract}

Keywords Enantioseparation, Chiral Mobile Phase Additive, (R,S)-Nadiflo xacin, $\beta$-Cyclodextrin

\section{Introduction}

In recent years, there has been considerable interest in the synthesis and separation of enantiomers of organic compounds especially because of their importance in biochemistry and pharmaceutical industry[1-5]. The method frequently used for separations, monitoring the progress of an asymmetric synthesis or optical purity of the product is chromatography with either liquids, gases or supercritical fluids as the mobile phase. Separation of the enantiomers comprising the racemate i.e. the resolution of the racemate is a common problem in stereochemical research as well as in the preparation of biologically active compounds, in particular drugs. One approach to separate enantiomers sometimes referred to as indirect enantiomeric resolution which involves the coupling of the enantiomers with an auxiliary chiral reagent to convert them in to diastereomers [6-11]. The diastereomers can be separated by any achiral separation technique[1]. The chirality of the enantiomeric molecules is caused by the presence of one or more chiral atom in their structure. The chirality sense and optical activity of the enantiomers are determined by their absolute configuration.

Reversed phase high performance liquid chromatography (RP-HPLC) techniques were used to separate and quantify

* Corresponding author:

sgaurav_thakur@rediffmail.com (Gaurav Singh)

Published online at http://journal.sapub.org/aac

Copyright @ 2012 Scientific \& Academic Publishing. All Rights Reserved enantiomers with high resolution. (S)-(-)-Nadifiloxacin[(s)-( -)-9-fluoro-6,7-dihydro-8-(4-hydro xypiperidino)-5-Methyl1-o xo-1 $\mathrm{H}, 5 \mathrm{H}$-benzo[i,j]quinolizine-2-carbo xy licacid] is a synthetic quinolone derivative with potent broad spectrum antibacterial activity and inhibits the enzyme DNA gyrase that is involved in the synthesis of bacterial DNA and its replication[12].<smiles>C[C@H]1CCc2c(N3CCC(O)CC3)c(F)cc3c(=O)c(C(=O)O)cn1c23</smiles>

Figure 1a. Chemical structure of S-Nadifloxacin<smiles>C[C@H]1CCc2c(N3CCC(O)CC3)c(F)cc3c(=O)c(C(=O)O)cn1c23</smiles>

Figure 1b. Chemical structure of R-Nadifloxacin 
Nadifloxacin is active against aerobic Gram-positive, Gram-negative and anaerobic bacteria including Propioniba cterium acnes and Staphylococcus epidermidi.The enantiomer compounds (fig-1a\&1b) vary greatly in their biological activity. As a result of it, the assessment of the biological activity of each enantiomer of a chiral molecule has been a standard practice in order to produce drugs and food products mainly as single enantiomers [13].

Cyclodextrin (CD) based chiral stationary phases (CSPs) or mobile phase additives are extensively applicable to separate enantiomers of large class of compounds [14-15].These are quite successful in separating the enantiomers of chiral molecules with aromatic substituents [16-20]. Consequently, cyclodextrin based CSPs are natural choice for addressing the liquid chro matographic chiral separation of these compounds .The determination of enantiomeric purity of the compound was, therefore, developed using cyclodextrin as a chiral selector. Because of low cost and unique physical characteristics, $\beta-C D$ is the most popularly used phase among the three phases of $C D$. A survey of literature reveals that an attempt has been made for the assay and quantification of R-(+) enantio mer impurity in the bulk drug which is L-arginine salt tetrahydrate of Nadifloxac in (WCK771) by HPLC using $\beta$-CD based CSPs [1]. However, enantiomeric resolution of Nadiflo xac in has not yet been carried out. A method has, therefore, been developed for the same with better resolution, lower limit of quantification (LOQ) and higher degree of reproducibility.

\section{Experimental}

\subsection{Instrumentation}

Chromatographic analysis is performed with waters 2695-separation module coupled with photodiode array detector (Compounds were detected at $\lambda-290 \mathrm{~nm}$ ) and column YMC C18 (250 mm x $3.0 \mathrm{~mm}) 3 \mu \mathrm{m}$ pore size $120 \check{\mathrm{A}}$ is used for chromatography. The oven temperature of HPLC was at $45^{\circ} \mathrm{C}$ and in jection volume is $20 \mu \mathrm{L}$. The flow rate of the mobile phase was adjusted at $1.5 \mathrm{~mL} \mathrm{~min}^{-1}$ and the total run time is $30 \mathrm{~min}$. Chromatographic data were controlled and processed on a computer running with millennium empower pro version 5.00.00.00.

\subsection{Chemicals}

Nadifloxacin is from Beijing Nine camp Medicine Technology Co.Ltd. Chaoyang (CHINA), Sodiu mhydro xide pellets, Triethylamine, di-sodium hydrogen orthophosphate (anhydrous) and Potassium di-hydrogen orthophos phate (all of AR grade); water and acetonitrile (HPLC grade) are from qualigens (India). Ethylene diaminetetraacetic acid (EDTA) disodium salt and $\beta$-Cyclodextrin (both of A R grade) from Across chemicals (India) were used during studies.

\subsection{Preparation of Stock Solution for Resolution}

$5.0 \mathrm{mg}$ of R-Nadifloxacin is weighed accurately and transferred into a standard $50 \mathrm{~mL}$ volumetric flask. It is thoroughly dissolved with $2 \mathrm{~mL}$ of $0.1 \mathrm{~N} \mathrm{NaOHsolution}$ and the volume was made up with diluent up to the mark and mixed thoroughly.

\subsection{Preparation of Solution for Resolution}

$50 \mathrm{mg}$ of S-Nadifloxacin is weighed accurately and transferred in to $25 \mathrm{~mL}$ volu metric flask. It is dissolved with $2 \mathrm{~mL}$ of $0.1 \mathrm{~N} \mathrm{NaOH}$ and $0.5 \mathrm{~mL}$ of resolution stock solution is added. The solution is now made up with diluent up to the mark and thoroughly mixed. It is then filtered through $0.45 \mu \mathrm{m}$ filter or finer porosity me mb rane filter.

\subsection{Preparation of Sample Solution}

$50 \mathrm{mg}$ of $\mathrm{S}$-Nadifloxacin is weighed accurately and transferred in to $25 \mathrm{~mL}$ volu metric flask. It is dissolved with $2 \mathrm{~mL}$ of $0.1 \mathrm{~N} \mathrm{NaOH}$. The solution is now made up with diluent up to the mark and thoroughly mixed. It is then filtered through $0.45 \mu \mathrm{m}$ filter or finer porosity me mbrane filter.

\subsection{Preparation of Buffer}

$1.0 \mathrm{~g}$ of Potassium di-hydrogen orthophosphate, $0.50 \mathrm{~g}$ of di-Sodium hydrogen orthophosphate (anhydrous), $50.0 \mathrm{mg}$ of E.D.T.A and $10.0 \mathrm{~g}$ cyclodextrin hydrate is dissolved in $1000 \mathrm{~mL}$ of water by constant stiring. It is then filtered through a $0.45 \mu \mathrm{m}$ filter or finer porosity me mb rane filter.

\subsection{Preparation of Mobile Phase}

A mixed degassed solution of buffer and acetonitrile $(\mathrm{ACN})$ in various ratios $(\mathrm{v} / \mathrm{v})$ is prepared and its $\mathrm{pH}$ is adjusted to the desired level with triethylamine. The mobile phase is also used as diluent.

\section{Results and Discussion}

Enantiomeric separation by HPLC is generally made by immobilizing the single enantiomers on to the stationary phase and resolution is the result of the formation of transient diastereomer by the interaction of CSPs. Enantiomer which form the most stable diastereomer is retained and opposite enantiomer forming less stable diastereomer will elute first. Interactions between CSPs and enantiomers are very week and require careful optimization by adjustment of the suitable mobile phase and temperature of the column to maximize the enantioselectivity. These interaction forces are ionic, $\pi-\pi$ interaction, hydrophobic effect and hydrogen bonding[6].

For cyclodextrin produced by the action of Bacillus macerans amy lase on starch, the size of CD depends upon the type of reaction between the two. CDs are the cyclic oligosaccharides containing six to twelve or eight D (+) glucopyranose units and bonded through alpha 1-4 linkages and form truncated conical cavity, the diameter of which depends upon the number of glucopyranose units. 
Commercially available CDs are pictured as hollow truncated cones, the torrodial structure having a hydrophilic surface. $\beta$-phase has seven units of glycopyranose the value ranging from $(6.0-8.0 \breve{A})$. $\beta$-CD has the widest application due to its pronounced kink shape whereas $\alpha-C D$ and $\gamma-C D$ are more planer. Its molecule has secondary -2 and -3 hydroxyl groups of the glycopyranose units lining at the mouth of the cavity and primary 6-hydroxyl groups at the rear of the cavity (fig-2).

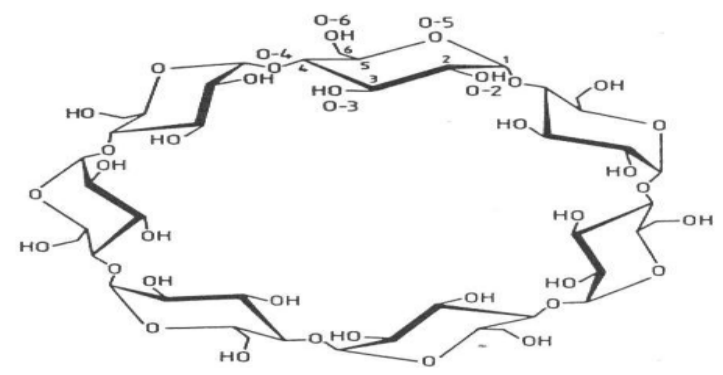

Figure 2. Structure of $\beta$-Cyclodextrin

This means that the cavity itself is a relatively hydrophobic region of the molecule and permits the inclusion of the hydrophobic portion.

The cavity is composed of the glucoside oxygen and methylene hydrogens giving it an apolar character. For the solute molecules, polar region of the molecules interact with the hydroxyl groups on the surface of cavity and provides the three point interaction[15,20,21]required for the chiral recognition.

The basic property of cyclodextrins that allow them to effect a large number of chemical separations is their ability to form selective inclusion complexes with a variety of guest molecules. The formation of this inclusion complex[22] may be caused by (a) A hydrophilic effect (b) Hydrogen bonding (c) Release of high energy water or modifier during complex formation (d) Combined effect of all these factors.

In general, binding to the cyclodextrin is governed by the molecule's ability to closely fit the cavity of the CD alongwith the polarity of the molecule itself. This fit depends both on size and shape of analyte concerning the CD cavity. For too small and too bulky molecules, there will be little or no binding at all. Larger molecules can be bound to $C D$, if certain groups or side chains of the molecule can penetrate the cavity effectively. The hydrophobic character of the cavity in a particular orientation is responsible for the stereoselective discrimination of chiral molecules that are fitted with in to the CD cavity during chro matography.

The enantiomeric separation due to chiral additive $(\beta-C D)$ in the mobile phase may involve any of the following general mechanis $\mathrm{m}$.

(i) Adsorption of the chiral selector to the solid phase in insitu formation of a temporary chiral stationary phase (CSP).

(ii) Stereoselectivecomplexation in the mobile phase.

(iii) Formation of the labile diastereo complexes with different distribution properties between the stationary and mobile phase.

However, the competition between mobile phase and stationary phase for $\beta$-CD plays a very important role. As studied earlier[23], a comparis on of column characteristics before and after the column is exposed to $\beta$-CD mobile phase, shows that column characteristics (retention time, efficiency, peak shape etc) are not changed when a $\beta$-CD modified mobile phase containing $10 \% \mathrm{ACN}$ is used. It shows that there is no adsorption of $\beta-\mathrm{CD}$ on to the stationary phase in case of $10 \%$ ACN solution used as the mobile phase. In the present case, this is most suitable mobile phase composition for enantio meric resolution of Nadiflo xac in (Table-1).

Table 1.Effect of mobile phase composition on USP resolution (rest parameters as per method)

\begin{tabular}{ccccc} 
& Ratio of & $\mathrm{pH}$ & Flow rate & USP \\
\cline { 2 - 5 } & Buffer : ACN & & (mL/min.) & Resolution \\
\hline 1 & $80: 20$ & 7.4 & 1.5 & 1.02 \\
2 & $85: 15$ & 7.4 & 1.5 & 1.12 \\
3 & $90: 10$ & 7.4 & 1.5 & 4.10 \\
\hline
\end{tabular}

The first important consideration to form a stable inclusion complex is proper fit of the molecule to the CD-cavity. As a general rule, substituted phenyl, naphthyl and biphenylrings can be included in the cavity of $\beta-\mathrm{CD}[24]$. Thus, the retention mechanis $m$ is a two equilibria process i.e. a reversible equilibrium of the solute in the bulk solvent mobile phase (a) with the stationary phase sites to from a complex and (b) with the $\beta-C D$ in the mobile phase to form an inclusion complex.

It has been observed earlier in case of L-arginine salt tetrahydrate of Nadiflo xac in that the analyte form inclusion complex with $\beta-C D$ and is stabilized by van der waal, hydrogen-bonding and hydrophobic interactions. The interactive selectivity is caused by the spatial difference between the isomers. $\beta-\mathrm{CD}$ is, therefore, used as chiral mobile phase additive (CMPA). The main advantage of this method is that the R-Nadifloxacin elutes first and avoids "smearing" under main co mpound peak[1].

Seeman et al[25] observed that analytes which form more stable inclusion complexes would be retained longer and be eluted later and those forming less stable complexes will be eluted earlier. S-Nadiflo xac in can enter the $\beta$-CD cavity to form an inclusion complex in the reversed phase mode which leads to the observed chiral separation. It forms relatively more stable inclusion complex and is eluted later. In addition to the solubility of $\beta-C D$ in the mobile phase used, the position of the substituents on the aromatic ring on one hand and the hydrophilic interaction between the $-\mathrm{OH}$ groups outside the cavity of $\beta-\mathrm{CD}$ on the other are the determining factors for the stability of inclusion complexes formed.

It is well established that, for a CD to form an enantioselectivediastereomeric complex, the substituents of the stereogeniccenter of analyte must be in close proximity to thesecondary-OH groups at the mouth of the $\mathrm{CD}$ in order to achieve the necessary three points of interaction $[15,20$, 
24]. If the portion of the molecule having stereogeniccenter resides in the cavity of $\mathrm{CD}$ upon inclusion, the stereogeniccenter will be buried inside the CD torus, not in the close pro ximity to the secondary-OH groups on the larger rig of the molecule. In this case, the substituent $\left(-\mathrm{CH}_{3}\right)$ on or near the analyte'sstereogeniccenter will be unable to interact with portion of the chiral selector that is most responsible for the chiral recognition. It can be concluded that when Nadifloxacin form an enantioselective inclusion complex with $\beta-C D$ in the reversed phase mode, their stereogeniccenter is located near the mouth of the $\mathrm{CD}$ selector.

However, the main driving force for the inclusion complex formation is through the release of enthalpy-rich water from the cavity due to the entrapping of guest molecules. Van der Waal forces, Hydrogen bonds and hydrophobic interactions help to keep the complex together. No covalent bonds are formed or broken during inclusion complex formation. Therefore, the inclusion complexation process can be considered as a replacement of water molecules with Nadifloxacin (Drug) molecules.

This view is supported by the explanation given by yang[26] for $\beta$-cyclodextrincomple xation and formu lation as an anti-HIV microbe.

In aqueous phase hydrophobic cavity of $\beta$-cyclodextrin is occupied by the water molecules, which is thermodynamically unfavorable. Therefore the water molecules have less tendency to form the hydrogen bonds in the same way as in the solutions and result in a higher enthalpy and higher energy.When hydrophobic guest molecules are incorporated in to the system, the energy of the system is lowered by substituting these enthalpy rich-water molecules with those of hydrophobic Nadiflo xacin (Drug) or guest molecules to form the complex of $\beta$-cyclodextrin and guest molecules [26].

Steric discrimination of the Nadifloxac in enantio mers can affect the formation of inclusion complex. Due to this discrimination S-Nadifloxacin will form more stable inclusion complex with $\beta$-cyclodextrin in comparison to R-Nadifloxacin. Therefore, S-Nadifloxacin will elute later and resolution takes place in between $\mathrm{R}$ and $\mathrm{S}$-Nadifloxacin .Thus, due to multifunctional mechanism derived from molecular inclusion and chemical interactions, the method is quite successful in the enantioseparation of Nadiflo xac in. The method is superior to the already reported one[1] for the enantioseparation of Nadiflo xac in because of better resolution, lower limit of quantification (LOQ) and higher degree of reproducibility.

\section{Optimization of the HPLC Enantioseparation}

The main features of the enantioseparation of racemic mixtu re of Nadifloxacin are given below.

Tables 1-3 shows the effect on the USP (United States Pharmacopeia) resolution of the variation in the composition of the mixture of buffer and acetonitrile alongwith variation in final $\mathrm{pH}$ value and the flow rate of the mobile phase.

Table 2. Effect of mobile phase pHon USP resolution (Rest parameters as per method)

\begin{tabular}{ccccc} 
& Ratio of & $\mathrm{pH}$ & Flow rate & USP \\
\cline { 2 - 5 } S.No. & Buffer :ACN & & $(\mathrm{mL} / \mathrm{min})$. & Resolution \\
\hline 1 & $90: 10$ & 7.0 & 1.5 & 1.02 \\
2 & $90: 10$ & 7.3 & 1.5 & 3.85 \\
3 & $90: 10$ & 7.4 & 1.5 & 4.10 \\
\hline
\end{tabular}

Table 3. Effect of flow rate of mobile phase on USP resolution(Rest parameters as per method

\begin{tabular}{ccccc}
$\mathrm{S}$ & Ratio of & $\mathrm{pH}$ & Flow rate & USP \\
\cline { 2 - 5 } $\mathrm{N}$. & Buffer : ACN & & $(\mathrm{mL} / \mathrm{min})$. & Resolution \\
\hline 1 & $90: 10$ & 7.4 & 1.8 & 3.45 \\
2 & $90: 10$ & 7.4 & 1.5 & 4.10 \\
3 & $90: 10$ & 7.4 & 2.2 & 2.28 \\
\hline
\end{tabular}

The extracted chromatogram of S-Nadifloxacin spiked with R-Nadifloxacin (Spiked at $0.1 \%$ level or $2 \mu \mathrm{g} \mathrm{mL}$ respectively with R-Nadifloxacin) is shown in figure-3 while that forS-Nadifloxacin is shown in figure-4. An excellent resolution $(\mathrm{Rs}=4.10)$ between the enantiomers and ideal peak shape with tailing factor 1.08 was obtained.

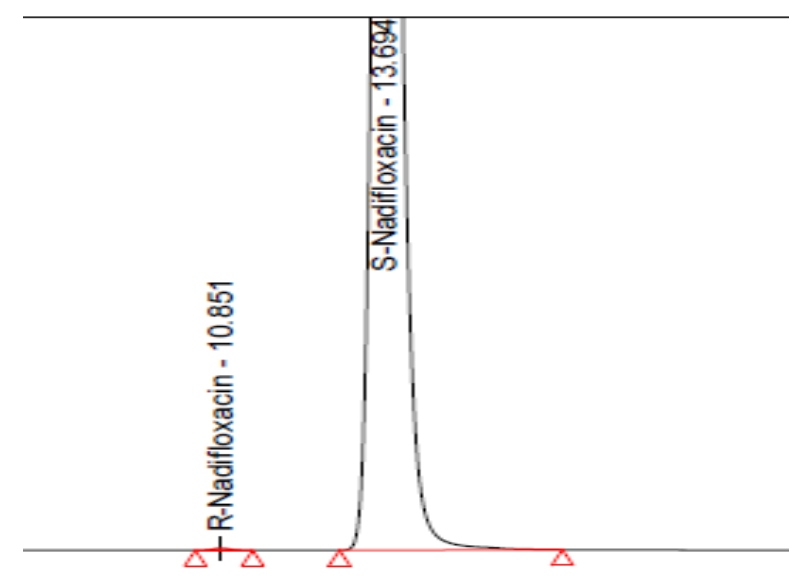

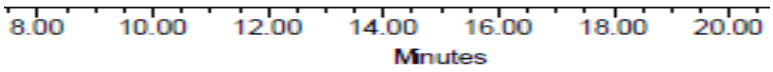

Retention time of R-Nadifloxacin: 10.851 , Area $\mu V^{*}$ Sec: 187976 , USPTailing: 1.08Retention time of S-Nadifloxacin: 13.694,

Area $\mu V^{*}$ Sec: 1498713117 ,USP Resolution : 4.10 ,USP Tailing: 1.23

Figure 3. Chromatogram of S-Nadifloxacin spiked with R-Nadifloxacin 


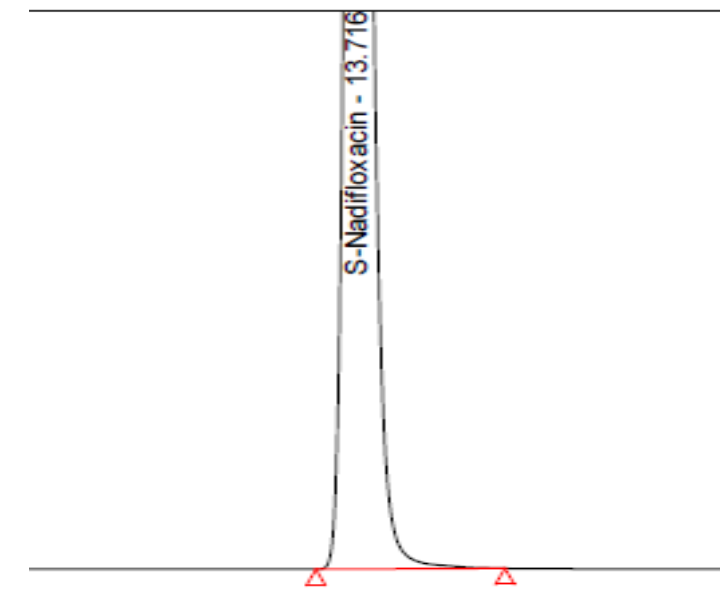

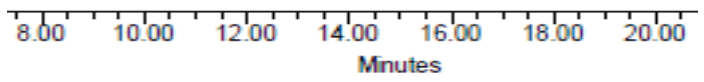

Retention time of S-Nadifloxacin: 13.716, Area $\mu \mathrm{V}^{*} \mathrm{Sec}$ : 1587713117 , USP Tailing: 1.22

Figure 4. Chromatogram of S-Nadifloxacin

\section{Validation of Analytical Method}

After the systematic optimization of the method the final conditions were found to be as follows: mobile phase (buffer and $\mathrm{ACN}$ ) ratio is $90: 10, \mathrm{pH} 7.4$, flow rate $1.5 \mathrm{~mL} \mathrm{~min}^{-1}$ and total run time is $30 \mathrm{~min}$.

\subsection{System Suitability}

Performance of the method was determined by injecting the resolution solution. The qualification criteria were resolution between two enantio mers which should be not less than 2.0 and tailing factor not more than 1.5, which ensures baseline separation and symmetrical peak shape for R-Nadiflo xacin.

\subsection{System Precision}

The System precision of the analytical method was determined by injecting the replicate injection of R-Nadiflo xacin with concentration $2 \mu \mathrm{g} \mathrm{mL} L^{-1}$.

\subsection{Method Precision}

Precision of the method was determined by the analysis of six spiked sample amount of R-Nadifloxacin at concentration of $2 \mu \mathrm{g} \mathrm{mL} \mathrm{m}^{-1}$ shown in (table -4).

\subsection{Ruggedness}

Ruggedness of the method was determined by the analys is of six spiked sample amount of R-Nadifloxacin at concentration of $2 \mu \mathrm{g} \mathrm{mL} \mathrm{m}^{-1}$ on different instrument and different colu mn shown in (table -4).

\subsection{Accuracy}

Accuracy of the method was confirmed by determining the $\%$ recovery of spiked amount ofR-Nadifloxacin at concentration 80,100 and $120 \%$ of $2 \mu \mathrm{g} \mathrm{mL} \mathrm{mL}^{-1}$ in thepre-analyzed sample of Nadifloxacin and is shown in (table -5).

Table 4. Ruggedness data for R-Nadifloxacin content

\begin{tabular}{ccc} 
& \multicolumn{2}{c}{ R-Nadifloxacin Content \% w/w } \\
\cline { 2 - 3 } & System-1 & System-2 \\
\hline Spiked Sample-1 & 0.102 & 0.101 \\
Spiked Sample-2 & 0.099 & 0.098 \\
Spiked Sample-3 & 0.101 & 0.101 \\
Spiked Sample-4 & 0.102 & 0.100 \\
Spiked Sample-5 & 0.100 & 0.100 \\
Spiked Sample-6 & 0.103 & 0.102 \\
Average & $\mathbf{0 . 1 0 1}$ & $\mathbf{0 . 1 0 0}$ \\
SD & $\mathbf{0 . 0 0 1}$ & $\mathbf{0 . 0 0 1}$ \\
\%RSD & $\mathbf{0 . 9 9}$ & $\mathbf{1 . 0 0}$ \\
O verall Average & & $\mathbf{0 . 1 0 1}$ \\
O verall SD & & $\mathbf{0 . 0 0 1}$ \\
Overall \%RSD & & $\mathbf{0 . 9 9}$ \\
\hline
\end{tabular}

Table 5. Recovery Results of R-Nadifloxacin

\begin{tabular}{ccccc}
\hline Amount & Amount & Amount & Amount & Recovery \\
\cline { 1 - 4 } Observed & Added & Present & Recovered & $\%$ \\
\cline { 1 - 4 }$\% \mathrm{w} / \mathrm{w}$ & $\% \mathrm{w} / \mathrm{w}$ & $\% \mathrm{w} / \mathrm{w}$ & $\% \mathrm{w} / \mathrm{w}$ & \\
\cline { 1 - 3 } 0.078 & 0.080 & 0.000 & 0.078 & 97.50 \\
0.079 & 0.080 & 0.000 & 0.079 & 98.75 \\
0.079 & 0.080 & 0.000 & 0.079 & 98.75 \\
0.102 & 0.100 & 0.000 & 0.102 & 102.00 \\
0.100 & 0.100 & 0.000 & 0.100 & 100.00 \\
0.099 & 0.100 & 0.000 & 0.099 & 99.00 \\
0.118 & 0.120 & 0.000 & 0.118 & 98.33 \\
0.118 & 0.120 & 0.000 & 0.118 & 98.33 \\
0.119 & 0.120 & 0.000 & 0.119 & 99.17 \\
& & & Avg. & 99.09 \\
\hline
\end{tabular}

\subsection{Linearity of R-Nadifloxacin}

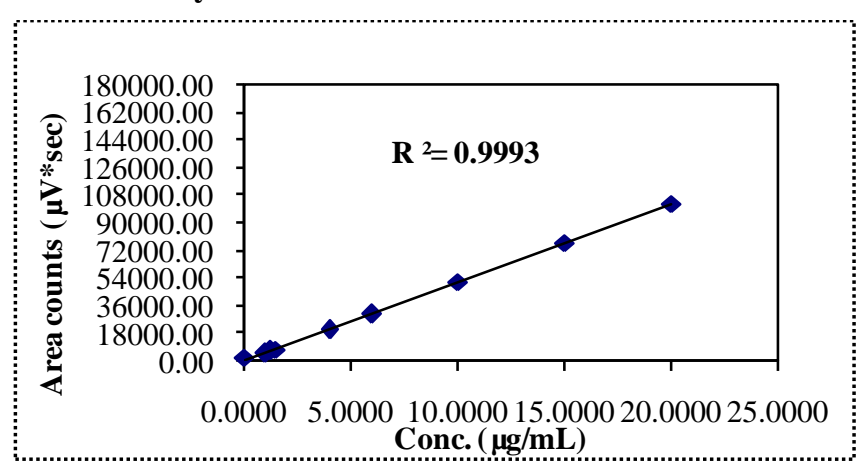

Figure 5. Linearity Curve of R-Nadifloxacin

The linearity of an analytical procedure is its ability (within a given range) to obtain test results which are directly proportional to the concentration (amount) of analyte in the sample. The Linearity of concentration vs peak area of $\mathrm{R}$-Nadifloxacin in this method over a concentration range of 
$0.040-20 \mu \mathrm{gL}^{-1}$ is shown by a straight line (fig-5).

\subsection{Limit of Detection and Quantification}

The limits of detection and quantification of R-Nadifloxacin in this method are summarized (table -6). As per Eurochem guidelines, the \% RSD of six replicate injection peak area should be not more than 10 for LOQ. In case of LOD, the \% RSD of six replicate in jection peak area should be not more than 33 . The limit of quantification and limit of detection for R-Nadifloxacin are $0.040 \mu \mathrm{g} \mathrm{mL}$ and0.020 $\mu \mathrm{g} \mathrm{mL}^{-1}$ respectively.

Table 6. LOD and LOQ of R-Nadifloxacin

\begin{tabular}{ccc}
\hline & $\begin{array}{c}\text { LOQ } \\
\text { Conc }(\mu \mathrm{g} / \mathrm{mL})\end{array}$ & $\begin{array}{c}\text { LOD } \\
\text { Conc }(\mu \mathrm{g} / \mathrm{mL})\end{array}$ \\
\cline { 2 - 3 } \# Injection & 0.040 & 0.020 \\
\cline { 2 - 3 } & & \\
1 & Area $\mu \mathrm{V}^{*} \mathrm{Sec}$ & Area $\mu \mathrm{V}^{*} \mathrm{Sec}$ \\
\hline 2 & 2569 & 1178 \\
3 & 2987 & 1198 \\
4 & 3143 & 1023 \\
5 & 3098 & 1675 \\
6 & 2576 & 1878 \\
Average & 2572 & 1564 \\
SD & 2824 & 1419 \\
$\%$ RSD & 281 & 335 \\
\hline
\end{tabular}

\section{Conclusions}

A simple, rapid and linear RP-HPLC method containing chiral mobile phase additive is given for the quantitative separation of R-Nadifloxacin from S-Nadifloxacin. The method is simple as it does not need any derivatization to diastereomers and economical too. It excludes the use of chiral stationary phase and uses very inexpensive $\beta$-cyclodextrin as chiral mobile phase additive and easily available RP-HPLC C18 colu mn for ch ro matography.

\section{ACKNOWLEDGEMENTS}

The authors thank Ankitasingh for editing the manuscript and organizing it for Web submission.

\section{REFERENCES}

[1] R.D. Yeole, A.S. Jadhav, K.R. Patil,V.P. Rane, M.L. Kubal, S. Singh ,M.V. Patel and H.F. Khorakiwala, "Validated chiral high-performance liquid chromatography method for a novel anti-methicillin-resistant staphylococcus aureusfluoroquinol one WCK 771", J. chromatogr. (A), Vol.1108, pp.38-42, 2006.
[2] T.Ooya, N.Kobayashi, T.Ichi, S. Sasaki and N.Yui, "Hydrogels having tubular $\alpha$-cy clodextrin structure: effect of nano-tube structure on long alkyl chain partitions", Science and Technology of Advanced Materials, Vol.4, pp. 39-42, 2003.

[3] E.Lipika, A.Selouane, D.Postel, C.Len, M.P.Vaccher, J-P.Bonte and C.Vaccher, "Enantioseparation of cis trans diastereomers of 2'3'-didehdro-2',3'dideoxy thiamidine analogs by high performance liquid chromatography and capilary electrophoresis", J.chromatogr. (A), vol.1034, pp. 161-167, 2004.

[4] L.Zhou, Bruce D.Johnson, C.Miller and Jean M.Wyvratt, "The separation of positional isomers by capillary electrochromatography",J. chromatogr. (A), vol. 875, pp. 389-401, 2000.

[5] D.D.Schumacher, C.R. Mitchell, T. Xiao, R.V.Rozhkov, R.C. Larock and D. W. Armstrong, "Determination of polyethylene glycol in low-density polyethylene by large volume injection temperature gradient packed capillary liquid chromatography", J. chromatogr. (A), Vol.1011, pp.37-47, 2003.

[6] J-Y Moon, H-J Jung, M. H. Moon, B.C. Chung and M.H. Choi, "Inclusion complex-based solid-phase extraction of steroidal compounds with entrapped $\beta$-cy clodextrin polymer", Steroids (A), Vol.73, pp.1090-1097, 2008.

[7] C-H Lin,W-R Fang,C-M Kuo,W-Y Chang, Y-C Liu ,W-Y Lin ,J-C Wu and C-E Lin, "Chiral separation of hydroxy flavanones in cyclodextrin-modified capillary zone electrophoresis using sulfated cyclodextrins as chiral selectors",J. chromatogr. (A), Vol.1188, pp.301-307, 2008.

[8] Y.Francois, A.Varenne, E. Juillerat, D.Villemin and P. Gareil, "Evaluation of chiral ionic liquids as additives to cyclodextrins for enantiomeric separations by capillary electrophoresis", J. chromatogr. (A), Vol.1155, pp.134-141, 2007.

[9] V.Cucinotta, A.Giuffrida, G.Grasso, G.Maccarrone, M.Messina and G.Vecchio, "High selectivity in new chiral separations of dansyl amino acids by cy clodextrin deriv atives in electrokinetic chromatography", J.chromatogr. (A), Vol.1155, pp.172-179, 2007.

[10] H-D Wang, L-Y Chu, H.Song, J.P.Yang, R. Xie and M. Yang, "Preparation and enantiomer separation characteristics of chitosan/ $\beta$-cyclodextrin composite membranes", J.Membrane Sc., Vol.297,pp-262-270, 2007.

[11] C. Jullian, S. Miranda, G. Zapata-Tarres, F. Mendizabal, C. Olea-Azar, "Studies of inclusion complexes of natural and modified cyclodextrin with (+) catechin by NMR and molecular modeling", Bioorganic \& Medicinal Chemistry, Vol.15, pp.3217-3224, 2007.

[12] P.Neoff, U, F.Haustein and N.Hittel, "Activity of Nadifloxacin (OPC-7251) and Seven Other Antimicrobial Agents against Aerobic and Anaerobic Gram-Positive Bacteria Isolated from Bacterial Skin Infections", Int. J. of Experimental and Clinical Chemotherapy, Vol.50,pp.196-198, 2004.

[13] United States food and drug administration, Chirality, Vol.4, pp-338-340, 1992.

[14] D.W.Armstrong and W.Demond, "Enantiomeric resolution of dansyl phenylalanine and analogs by microcolumn liquid 
chromatography with $\beta$-cyclodextrin as mobile phase additive", J .Chromatogr.Sc., Vol.22, pp.411-415, 1984.

[15] D.W.Armstrong, T.J.Word, R.D.ArmstrongandT.F.Beesley, "Separation of drug stereoisomers by the formation of beta-cyclodextrin inclusion complexes", Science, Vol.232, pp.1132-1142. 1986.

[16] C.R.Mitchell, M.Desai, R.Mcculla, W.Jenks and D.W.Armstr ong, "Use of Native and DerivatizedCyclodextrin Chiral Stationary Phases for the Enantioseparation of Aromatic and Aliphatic Sulfoxides by High Performance Liquid Chromatography", Chromatographia, Vol.56, pp.127-132, 2002.

[17] D.W.Armstrong and J.Jukoski, "Direct enantiomeric resolution of monoterpene hydrocarbons via reversed-phase high-performance liquid chromatography with an $\alpha$-cyclodextrin bonded stationary phase", J.Chromatogr. (A), Vol.666, pp.445-448, 1994.

[18] D.W.Armstrong, T.L.Ward, A.Czech, B.P.Czech and R.A.Bastsch., "Synthesis, rapid resolution, and determination of absolute configuration of racemic 2,2'-binaphthyldiyl crown ethers and analogs via .beta.-cyclodextrincomplexatio n”, J.Org.Chem., Vol.50, pp.5556-5559, 1985.

[19] D.W.Armstrong, L.W.Chang, S.C.Chang, X.Wang, H.Ibrahim, G.R.Reid and T.E.Beesley, "Comparison of the Enantioselectivity of $\beta$-Cyclodextrin vs. Heptakis-2,3-O-dim ethyl- $\beta$-cyclodextrin LC Stationary Phases Comparison of the Enantioselectivity of $\beta$-Cy clodextrin vs. Heptakis-2,3-O-dim ethyl- $\beta$-cyclodextrin LC Stationary Phases", J.Liq.
Chromatogr.Rel.Technol, Vol.20, pp.3279-3295, 1997.

[20] D.W.Armstrong, W.Demond and B.P.Czech., "Separation of Metallocene En antiomers by Liquid Chromatography Chiral Recognition Via Cyclodextrin Bonded Phases", Anal chem., Vol.57,pp.481-484, 1985.

[21] M.L. Bender and M.Komiyama, cyclodextrin chemistry, Springer, Berlin. 1978.

[22] J.J. Tang, Dissertation, Seton Hall University, N.J., 1996.

[23] J.J. Tang and L.J.Clinelove, "Formation constants of polynuclear aromatic compounds and $\beta$-cyclodextrin inclusion complexes in $\beta$-cyclodextrin modified mobile phase high performance liquid chromatography system", Anal.chim.Acta, Vol.344, pp.137-143, 1997.

[24] J.I.Seeman, H.V.Secor, D.W. Armstrong, K.D.Timmons and T.J. Ward, "Enantiomeric resolution and chiral recognition of racemic nicotine and nicotine analogs by .beta.-cyclodextrin complexation. Structure-enantiomeric resolution relationships in host-guest interactions", Anal.chem., Vol.60, pp-2120-2127, 1988.

[25] W.L. Hinze, T.E Riehl, D.W.Armstrong, W.Demond, A.Alak and T.J.Ward, "Liquid chromatographic separation of enantiomers using a chiral beta.-cyclodextrin-bonded stationary phase and conventional aqueous-organic mobile phases", Anal.Chem., Vol.57,pp-237-242, 1986.

[26] H.Yang, Ph.D Thesis, University of Pitts burgh., pp.33-34, 2008. 